ARTÍCULO ORIGINAL

\title{
Brotes de babesiosis y anaplasmosis bovina diagnosticados en el INTA Salta, Argentina: período 2006-2016
}

\author{
Araoz $\mathrm{V}^{1^{*}}$, Micheloud $\mathrm{JF}^{2,3}$, Gaido $\mathrm{AB}^{2}$, Salatin $\mathrm{AO}^{2}$, Aguirre $\mathrm{DH}^{2}$ \\ ${ }^{1}$ Instituto Nacional de Investigación Agropecuaria (INIA), Colonia, Uruguay \\ ${ }^{2}$ Instituto Nacional de Tecnología Agropecuaria (INTA) - CIAP - Instituto de Investigación Animal \\ del Chaco Semiárido - Área de investigación en Salud Animal - EEA Salta (Cerrillos), Argentina. \\ ${ }^{3}$ Facultad de Ciencias Agrarias y Veterinarias, Universidad Católica de Salta, Argentina
}

* Correspondencia: V Araoz. INIA, Ruta 50 - km 11, (70000) La Estanzuela, Colonia, Uruguay.

E-mail: varaoz@inia.org.uy

Recibido: 27 Noviembre 2017. Aceptado: 20 Diciembre 2017. Disponible en línea: 27 Diciembre 2017

Editor: S. Nava

RESUMEN. Se realizó un estudio retrospectivo de brotes de babesiosis y anaplasmosis (tristeza parasitaria bovina, TPB) ocurridos entre 2006 y 2016 en el Noroeste argentino (NOA) para evaluar algunos aspectos epidemiológicos y clínicos de estas enfermedades. De 4.398 muestras para diagnóstico, $238(5,4 \%)$ tuvieron sospechas de TPB, las cuales se confirmaron en $106(44,3 \%)$ casos por observación microscópica de los agentes causales. Cuarenta y siete (44,3\%) brotes se debieron a Anaplasma marginale, $40(37,7 \%)$ a Babesia bovis y $14(13,2 \%)$ a Babesia bigemina. En cinco $(4,7 \%)$ focos de babesiosis no se determinó la especie causal de Babesia. Los brotes se diagnosticaron en 62 establecimientos ganaderos de cuatro provincias (Salta, Jujuy, Santiago del Estero y Chaco). La mayoría ocurrieron en verano y otoño, con máxima incidencia en abril y nula en setiembre para ambas noxas. Las vacas adultas fueron la categoría de ganado más afectada. En 58\% (61/106) de los brotes murió al menos un bovino, con un número promedio de cuatro muertes por brote. Se concluye que la TPB continúa incidiendo negativamente en la producción bovina del NOA.

SUMMARY. Bovine babesiosis and anaplasmosis outbreaks diagnosed at INTA Salta, Argentina: 2006-2016. A retrospective study of babesiosis and anaplasmosis outbreaks (tick fever, TF) was made, which occurred from 2006 to 2016 in Northwest Argentina (NWA), to evaluate clinical and epidemiological aspects of these diseases. From 4,398 specimens, 238 (5.4\%) were associated with suspicions of TF and 106 of these cases (44.3\%) were confirmed as TF outbreaks by microscopic observation of causal agents. Forty seven (44.3\%) were due to Anaplasma marginale, $40(37.7 \%)$ to Babesia bovis and $14(13.2 \%)$ to Babesia bigemina. In five $(4.7 \%)$ outbreaks of babesiosis, Babesia species were not determined. Outbreaks were diagnosed at 62 different cattle ranches in four provinces (Salta, Jujuy, Santiago del Estero and Chaco). The outbreaks occurred mostly during summer and autumn with a peak of incidence in April and null incidence in September for both diseases. Adult cows were the most affected cattle category. At least one death was recorded in $58 \%(61 / 106)$ of the outbreaks and the average number of deaths was determined as four by outbreak. We conclude that TF maintain its negative historical impact on cattle production of NWA.

Palabras clave: hemoparásitos, enfermedades transmitidas por garrapatas, casuística, epidemiología

Key words: hemoparasites, tick borne disease, cases description, epidemiology

\section{Introducción}

Las babesiosis (Babesia bovis y Babesia bigemina) y la anaplasmosis (Anaplasma marginale) de los bovinos son enfermedades anemizantes que integran el complejo nosológico conocido como 'Tristeza parasitaria bovina' (TPB) en el Cono Sur americano (Späth, 1986; Begeres de Almeida et al., 2006; Solari et al., 2013). Este representa uno de los principales problemas sanitarios que afectan a rodeos de cría y lecheros del Noroeste argentino (NOA) (Späth, 1986; Guglielmone et al., 1992a; Aguirre et al., 2011). La muerte de animales, abortos, costo de tratamientos y honorarios profesionales (Guglielmone et al., 1992a; Aguirre et al., 2011), 
así como la merma en los índices productivos, son las principales causas de pérdidas económicas por estas noxas.

Los tres hemoparásitos son transmitidas por vectores, siendo Rhipicephalus (Boophilus) microplus el vector exclusivo para ambas Babesia (Guglielmone, 1995), cuyos rasgos epidemiológicos obedecen al ciclo biológico de dicho ixódido (Mastropaolo et. al., 2017). Por su parte, A. marginale puede ser transmitido biológicamente por esta u otras garrapatas (Aguirre et al., 1994; Gaido et al., 1995) pero también en forma mecánica por dípteros hematófagos (géneros Tabanus, Stomoxys y varias especies de mosquitos) y de manera iatrogénica por fómites (Kocan et al., 2010). La distribución estacional de brotes de TPB en Argentina presenta similitudes entre diferentes regiones geográficas y con países vecinos (Guglielmone et al., 1997; Sarmiento y Zimmer, 2010; Begeres de Almeida et al., 2006; Solari et al., 2013). Aunque todos suelen mostrar una marcada variabilidad interanual, principalmente como resultado de factores medioambientales y de manejo que modulan la interacción entre vectores y bovinos (Nava et al., 2013). Un estudio serológico bastante reciente refleja la existencia de amplias áreas del NOA enzoóticamente inestables para ambas Babesia y $A$. marginale, con alto riesgo de ocurrencia de brotes severos de TPB (Mangold y Mastropaolo, 2013).

Para la región NOA de Argentina, existe un solo estudio descriptivo de brotes de TPB en rodeos lecheros de un área geográfica restringida (Valle de Lerma, Salta) (Späth, 1986). En este trabajo se presenta la casuística de TPB de una zona más amplia, diagnosticada en el Área de Investigación en Salud Animal (AISA) del INTA Salta durante un periodo de 11 años, con el objetivo de describir aspectos epidemiológicos y clínicos de los brotes.

\section{Materiales y Métodos}

Se revisó la totalidad de protocolos ingresados al AISAINTA Salta durante el periodo comprendido entre enero de 2006 y diciembre de 2016 y se seleccionaron aquellos vinculados con el complejo TPB. Los casos se diagnosticaron a partir de muestras de 675 bovinos, provenientes de 62 establecimientos ganaderos, ubicados entre latitudes $22^{\circ}-27^{\circ} \mathrm{S}$ y longitudes $63^{\circ}-66^{\circ} \mathrm{W}$. El tipo de muestras recibidas en casos de sospecha de TPB fueron: extendidos de sangre $(84,5 \%)$, sangre entera con anticoagulante $(18,5 \%)$ y muestras de tejidos (orejas y cola: $12,2 \%$, sistema nervioso central: $3,8 \%$ y otros: $5,9 \%)$. En este último caso se realizaron improntas y extendidos de los órganos en portaobjetos. El material procesado fue fijado con metanol y coloreado con Giemsa al $10 \%$ durante 30 minutos, para su posterior examen con microscopio óptico en inmersión $(1000 x)$. En los casos en que se recibió sangre con anticoagulante, se determinó el índice hematocrito.
Para el diagnóstico se siguió el criterio propuesto por Benavides Ortiz et al. (2012), quienes tomaron a la proporción de eritrocitos infectados en extendidos finos de sangre periférica como el principal parámetro para confirmar un caso positivo, aunque datos complementarios como el hematocrito y hallazgos clínicos y de necropsia (temperatura rectal, debilidad, ictericia, hemoglobinuria, esplenomegalia) también fueron considerados. En los casos de babesiosis la identificación de la especie causal se realizó evaluando la morfología de los hemoparásitos. Se definió como brote la ocurrencia en un mismo establecimiento de uno o más casos clíni$\cos u$ óbitos durante un breve lapso ( $\leq$ dos meses), con diagnóstico confirmado en laboratorio (Späth, 1986). Para cada brote se evaluó el agente causal responsable, la fecha de presentación, la ubicación geográfica del brote, el sistema productivo y la categoría y raza de bovinos involucrados. En los casos en que la información se encontraba detallada en el protocolo de ingreso, se evaluaron los signos clínicos, el grado de anemia y la ocurrencia de muertes. Dado que no se hizo un seguimiento de cada brote no fue posible calcular las tasas de morbi-mortalidad y letalidad en cada uno de ellos.

\section{Resultados}

Del total de protocolos registrados en el periodo de estudio ( $n=4398), 238(5,4 \%)$ estuvieron relacionados con hemoparasitosis, de los cuales $106(44,6 \%)$ se confirmaron como brotes de TPB. Resultaron negativos 120 casos $(50,4 \%)$ y en 12 casos $(5 \%)$ no se logró arribar al diagnóstico por mala calidad de las muestras.

De los brotes confirmados, en 47 (44,3\%) se identificó como agente causal a $A$. marginale, en $40(37,7 \%)$ a $B$. bovis, en $14(13,2 \%)$ a $B$. bigemina y en cinco $(4,7 \%)$ a Babesia sin identificación de la especie. En nueve de los brotes se identificaron dos agentes en forma simultánea, con cinco casos de $A$. marginale $+B$. bovis, tres de $A$. marginale $+B$. bigemina y solo uno de $B$. bovis + B. bigemina.

La casuística varió ampliamente entre años, con un mínimo en 2010, cuando se diagnosticó un único brote (B. bigemina) y máximos durante 2007 y 2016, con 16 y 25 brotes, respectivamente (Figura 1). En cuanto a la estacionalidad, el $72 \%$ de los brotes del período estudiado se produjo en la primera mitad del año con un pico en abril. La casuística otoñal (abril-junio) fue ligeramente superior a la estival (enero-marzo): $42 \%$ versus $30 \%$. Los brotes restantes (28\%) ocurrieron en la segunda mitad del año, exceptuando septiembre, mes en el cual no se registraron brotes de TPB durante todo el período evaluado (Figura 2).

En cuanto a la distribución geográfica, 97 (91,5\%) brotes ocurrieron en 13 departamentos de la provincia de Salta, cinco brotes $(4,7 \%)$ en tres departamentos de Jujuy y dos pares de brotes $(3,8 \%)$ ocurrieron, 
respectivamente, en las provincias de Santiago del Estero y Chaco (Figura 3). Más de la mitad (57\%) de la casuística salteña se produjo en el área central de la provincia, sobresaliendo el departamento Chicoana (Ecorregión: Valles templados) con 26 brotes. Dos de los brotes de babesiosis registrados en Jujuy ocurrieron en zona de Prepuna (departamento Tumbaya), inapropiada para el establecimiento de la garrapata vector, a la cual llegó por el traslado de bovinos infestados desde áreas favorables para el desarrollo de su ciclo (Marín y Aguirre, 2017).

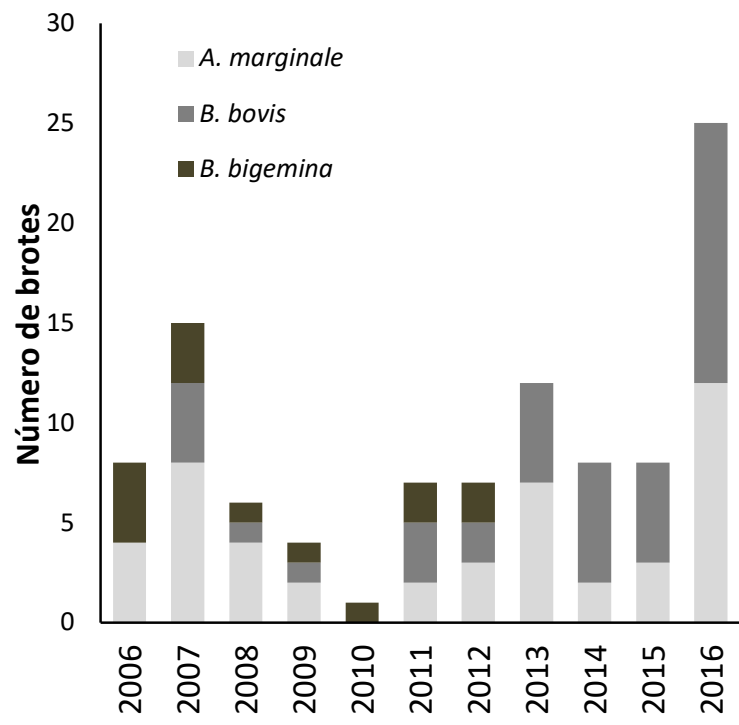

Figura 1: Distribución anual y por agente etiológico de brotes de 'Tristeza parasitaria bovina' diagnosticados en el AISA-INTA Salta (periodo 2006-2016).

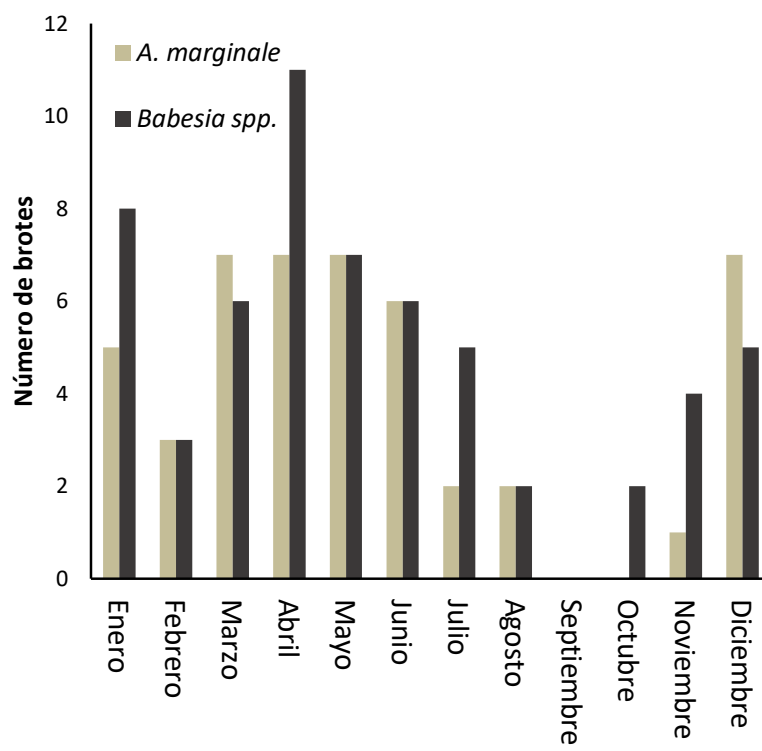

Figura 2: Ocurrencia mensual acumulada de brotes de 'Tristeza parasitaria bovina' diagnosticados en el AISA-INTA Salta (periodo 2006-2016).

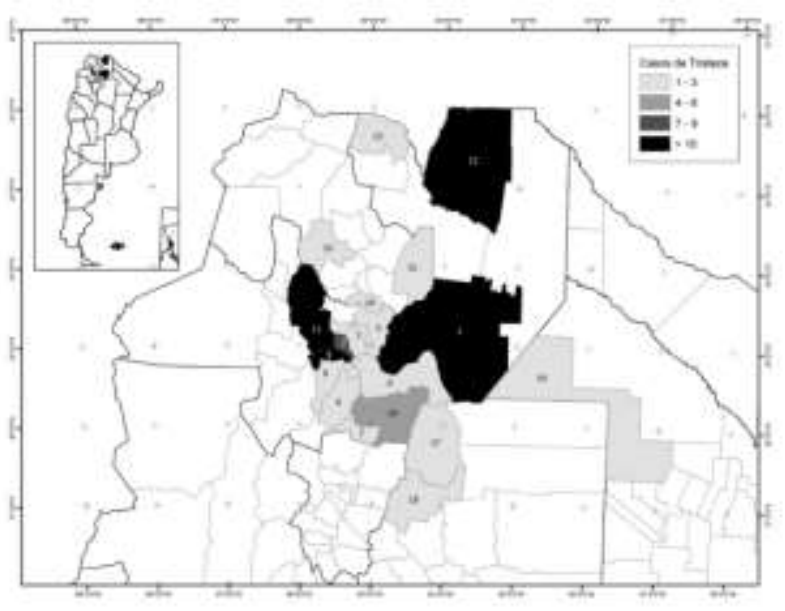

Figura 3: Mapa del Noroeste argentino mostrando abundancia de brotes de 'Tristeza parasitaria bovina' por Departamento en cuatro provincias (período 2006-2016). Salta: (1) Anta, (2) Capital, (3) Cerrillos, (4) Chicoana, (5) Gral. Güemes, (6) Guachipas, (7) La Candelaria, (8) La Viña, (9) Metán, (10) Rosario de la Frontera, (11) Rosario de Lerma, (12) Gral. San Martín, (13) Santa Victoria; Jujuy: (14) El Carmen, (15) Santa Bárbara, (16) Tumbaya; Santiago del Estero: (17) Gral. Pellegrini, (18) Jiménez; Chaco: (19) Alte. Brown.

La distribución de brotes según sistema productivo y agente causal se muestra en la Tabla 1, apreciándose neto predominio de la casuística en el sistema de cría, seguido por el de engorde. Considerando la principal categoría de bovinos afectada en cada brote, las vacas adultas (> 2,5 años) lideraron la casuística con un $41 \%$ de los casos, seguidas por los novillos y novillitos (28\%), vaquillonas $(14 \%)$, toros y toritos $(12 \%)$ y terneros $(5 \%)$. Los biotipos vacunos más involucrados fueron las razas sintéticas Brangus y Braford (88 brotes), seguidas por Holando-Argentino (11 brotes) y Aberdeen Angus (siete brotes). La distribución de los brotes a lo largo del año fue similar en todos los sistemas productivos.

Tabla 1: Brotes de 'Tristeza parasitaria bovina' y su distribución porcentual según sistema productivo diagnosticados en el AISA-INTA Salta. Periodo 2006-2016.

\begin{tabular}{|c|c|c|c|c|c|c|c|c|}
\hline \multirow{3}{*}{ Agente } & \multicolumn{8}{|c|}{ Sistema productivo } \\
\hline & \multicolumn{2}{|c|}{ Cría } & \multicolumn{2}{|c|}{ Engorde } & \multicolumn{2}{|c|}{ Tambo } & \multicolumn{2}{|c|}{ Recría } \\
\hline & $\mathrm{n}$ & $\%$ & $\mathrm{n}$ & $\%$ & $\mathrm{n}$ & $\%$ & $\mathrm{n}$ & $\%$ \\
\hline A.marginale & 25 & 38 & 11 & 42 & 9 & 82 & 2 & 50 \\
\hline B.bovis & 25 & 38 & 13 & 50 & 1 & 9 & 1 & 25 \\
\hline B.bigemina & 10 & 15 & 2 & 8 & 1 & 9 & 1 & 25 \\
\hline Babesia sp. & 5 & 8 & 0 & 0 & 0 & 0 & 0 & 0 \\
\hline Total & 65 & 61 & 26 & 25 & 11 & 10 & 4 & 4 \\
\hline
\end{tabular}

En el 93\% (99/106) de los brotes confirmados el profesional/productor informante sospechó de TPB. En 61 $(57,5 \%)$ de los 106 brotes se registró al menos un bovino muerto, con un máximo de 54 óbitos durante un brote de anaplasmosis en Jujuy (Aguirre et al., 2011). El número promedio de muertes por brote de TPB fue de cuatro bovinos. Además, en diez de los brotes ocurrieron abortos como evento asociado, con un máximo de 21 abortos en un brote de babesiosis ( $B$. bovis) en Santiago del Estero. La anemia fue también un rasgo 
destacado en los bovinos afectados, pues sólo en cuatro $(10,2 \%)$ de 39 brotes en los que se determinó el hematocrito se registraron valores medios superiores al umbral eritrocitario considerado normal $(\geq 35 \%)$ para bovinos adultos cruza índica.

\section{Discusión}

La amplia mayoría de los brotes se produjo en la provincia de Salta, particularmente en el área central, sede del AISA del INTA. Esta se localiza en el Valle de Lerma, zona agrícola-ganadera con relativo predominio de explotaciones lecheras y de engorde. De tal modo, todos los brotes registrados en rodeos lecheros $(n=11)$ correspondieron a esta zona, al igual que en un estudio anterior (Späth, 1986). Aun así, la elevada casuística en el área central de Salta no se interpreta resultante de una mayor incidencia de TPB, sino como producto de la cercanía de los rodeos afectados al centro de estudio (AISA). La vaca de cría adulta fue la más frecuentemente afectada por TPB. Esto era esperado dado que las vacas representan la categoría más numerosa del sistema productivo ganadero predominante en el NOA: la cría bovina. Estudios previos en la misma u otras áreas coincidieron con esta mayor casuística en bovinos adultos (Späth, 1986; Begeres de Almeida et al., 2006). Es conocido a su vez que tanto las babesiosis como la anaplasmosis son enfermedades que afectan particularmente al ganado adulto carente de inmunidad (Guglielmone et al., 1992a; Mangold y Mastropaolo, 2013), aunque según nuestros resultados los terneros no están exentos de padecer cuadros clínicos de TPB. La mayor incidencia en las cruzas índicas Brangus y Braford era también esperada por ser los biotipos más utilizados en los sistemas productivos de carne predominantes en el NOA. Si bien estas razas son reconocidas como más resistentes a $R$. (B.) microplus que las razas europeas (Guglielmone et al., 1992b), resultan igualmente susceptibles a las babesiosis y la anaplasmosis (Bock et al., 1999a; 1999b).

Los aspectos epidemiológicos de la casuística de TPB fueron similares a los descriptos previamente para rodeos lecheros del Valle de Lerma (Späth, 1986). En efecto, sobre 172 brotes, dicho autor informó proporciones casi idénticas a las actuales de anaplasmosis $(44,7 \%)$ respecto de las babesiosis $(55,3 \%)$. En relación con la estacionalidad, el $91 \%$ de los brotes de babesiosis ocurrió entre noviembre y junio, mientras el $89 \%$ de los de anaplasmosis se produjo entre enero y julio, con un pico en abril para ambos agentes (Späth, 1986). Acorde con nuestros datos este autor registró al trimestre agosto-octubre como el de menor cantidad de brotes de TPB. Este bache en la casuística se explicaría al menos para las babesiosis- por la nula eclosión de huevos de $R$. microplus durante el invierno, señalada recientemente para distintos sitios del NOA (Canevari et al., 2016; Mastropaolo et al., 2017). A su vez, el predominio de $A$. marginale y $B$. bovis aquí mencionado coincide también con lo descripto por Sarmiento y Zimmer
(2010) para brotes ocurridos en la provincia de Corrientes durante un breve lapso (2009-2010). Por otra parte, en la provincia de Santa Fe se informó una estacionalidad similar para brotes de anaplasmosis en rodeos de cría, pero no así en rodeos lecheros, donde ellos ocurrieron sin estacionalidad definida (Guglielmone et al., 1997). En nuestro estudio el número de casos en rodeos lecheros fue insuficiente para establecer patrones de estacionalidad.

Los resultados del presente trabajo tampoco difieren sustancialmente de estudios retrospectivos originados en países vecinos del Cono Sur. Uno de ellos, realizado en Brasil, evaluó la casuística de TPB recibida en un Laboratorio estatal (Pelotas, Rio Grande do Sul) durante 28 años (periodo 1978-2005). Tal como ocurrió aquí, el $79,1 \%$ de los brotes $(n=221)$ se produjo en la primera mitad del año, con incidencia apenas superior en otoño $(40,6 \%)$ que en verano $(38,5 \%)$, siendo abril y mayo los meses con mayor registro de brotes (42\%) (Begeres de Almeida et al., 2006). La menor casuística de TPB en Brasil coincidió también con el mes de septiembre. Igualmente, los brotes de babesiosis $(60,9 \%)$ superaron a los de anaplasmosis $(29,1 \%)$ si bien, a diferencia de nuestros datos, $B$. bovis predominó como agente individual frente a $A$. marginale. Otro informe generado en Uruguay sobre la casuística de tres Laboratorios regionales durante 24 años (periodo 1988-2011) arrojó resultados similares. En efecto, el $68,8 \%$ de los brotes ( $n=$ 300 ) en dicho país ocurrió en la primera mitad del año, con incidencia ligeramente superior en otoño $(35,3 \%)$ que en verano $(33,5 \%)$, resultando marzo y abril los meses con mayor registro de brotes (31\%) (Solari et al., 2013). Por su parte, los brotes de babesiosis $(66,1 \%)$ duplicaron a los de anaplasmosis $(33,9 \%)$, prevaleciendo también aquí $B$. bovis sobre a $A$. marginale (Solari et al., 2013). Por último, la mortalidad media en estos estudios fue similar o superior a la registrada por nosotros: $4,5 \%$ en Uruguay (Solari et al., 2013) y $6,8 \%$ en Brasil (Begeres de Almeida et al., 2006).

Tanto en nuestro análisis como en todos los antecedentes bibliográficos, la mayor parte de los brotes se debió a un único agente causal. Las infecciones asociadas representaron una escasa proporción $(8,5 \%)$ de los casos y en todas ellas pudo identificarse un patógeno principal responsable del cuadro clínico.

En línea con informes previos (Späth, 1986; Guglielmone et al., 1992a; Aguirre et al., 2011) se concluye que las hemoparasitosis siguen impactando negativamente en la producción bovina del NOA, pese a que desde hace varias décadas se dispone de tecnología para su control (Mangold y Mastropaolo, 2013). Las muertes registradas en más de la mitad de los brotes son apenas un indicador, si bien el más importante (Aguirre et al., 2011), de las cuantiosas pérdidas que estas noxas provocan año tras año en la ganadería bovina regional. 


\section{Agradecimientos}

Los autores agradecen al Lic. RRNN José N. Volante por la confección del mapa coroplético. El trabajo se realizó con recursos del CIAP- IIACS- INTA Salta, en el marco de una Beca de Formación otorgada a la Médico Veterinaria Virginia Aráoz durante el periodo 2015-2016.

\section{Bibliografía}

Aguirre DH, Gaido AB, Viñabal AE, Echaide ST de, Guglielmone AA. 1994. Transmission of Anaplasma marginale with adult Boophilus microplus ticks fed as nymphs on calves with different levels of rickettsaemia. Parasite 1: 405-407.

Aguirre DH, Neumann RD, Torioni de Echaide S, Mangold AJ. 2011. Pérdidas económicas directas por un brote de anaplasmosis en un rodeo de cría del noroeste argentino. Rev. Arg. Prod. Anim. 31: 145-153.

Begeres de Almeida M, Tortelli FP, Riet-Correa B, Montiel Ferreira JL, Soares MP, Farias NAR, Riet-Correa F, Schild AL. 2006. Tristeza parasitaria bovina na regiao sul do Rio Grande do Sul: estudo retrospectivo de 1978-2005. Pesq. Vet. Bras. 26: 237-242.

Benavides Ortiz E, Polanco Palencia N, Vizcaíno Gerdts O, Betancur Hurtado O. 2012. Criterios y protocolos para el diagnóstico de hemoparásitos en bovinos. Rev. Cienc. Anim. 5: 3149.

Bock RE, Kingston TG, De Vos AJ. 1999a. Effect of breed cattle on transmission rate and innate resistance to infection with Babesia bovis and B. bigemina transmitted by Boophilus microplus. Aust. Vet. J. 77: 461-464.

Bock RE, Kingston TG, De Vos AJ. 1999b. Effec of breed of cattle on innate resistance to infection with Anaplasma marginale transmitted by Boophilus microplus. Aust. Vet. J. 77 (11): 748-751.

Canevari JT, Mangold AJ, Guglielmone AA, Nava S. 2016. Population dynamics of the cattle tick Rhipicephalus (Boophilus) microplus in a subtropical subhumid region of Argentina for use in the design of control strategies. Med. Vet. Entomol. 31: 6-14.

Gaido AB, Viñabal AE, Aguirre DH, Echaide ST de, Guglielmone AA. 1995. Transmission of Anaplasma marginale by the threehost tick Amblyomma neumanni in the laboratory. Folia Parasitol. 42: 72.

Guglielmone AA. 1995. Epidemiology of babesiosis and anaplasmosis in South and Central America. Vet. Parasitol. 57: 109-119.

Guglielmone AA, Abdala AA, Anziani O, Mangold AJ, Volpogni MM, Vanzini VR. 1997. Different seasonal occurrence of anaplasmosis outbreaks in beef and dairy cattle in an area of Argentina free of Boophilus microplus ticks. Vet. Quart. 19: 3233.

Guglielmone AA, Aguirre DH, Späth EJA, Gaido AB, Mangold AJ, de Ríos LG. 1992a. Long-term study of incidence and financial loss due to cattle babesiosis in an Argentinian dairy farm. Prev. Vet. Med. 12: 307-312.

Guglielmone AA, Mangold AJ, Gaido AB, Aguirre DH. 1992b. Natural parasitism with Boophilus microplus (Acari: Ixodidae) in cattle biotypes with different proportions of Bos taurus (Spanish and British) and Bos indicus genes. Res. Rev. Parasitol. 52: 77-82.

Kocan KM, de la Fuente J, Blouin EF, Coetzee JF, Ewing SA. 2010. The natural history of Anaplasma marginale. Vet. Parasitol. 167: 95-107.

Mangold AJ, Mastropaolo M. 2013. Epidemiología y control de hemoparásitos (Babesia y Anaplasma) en Argentina. En: Fiel C, Nari A (Coord.). Enfermedades Parasitarias de Importancia Clínica y Productiva en Rumiantes. Fundamentos epidemiológicos para su diagnóstico y control. Ed. Hemisferio Sur S.R.L, Montevideo. pp. 639-655.

Mastropaolo M, Mangold AJ, Guglielmone AA, Nava S. 2017. Non-parasitic life cycle of the cattle tick Rhipicephalus (Boophilus) microplus in Panicum maximum pastures in northern Argentina. Res. Vet. Sci. 115: 138-145.

Marín RE, Aguirre DH. Descripción de dos brotes de babesiosis nerviosa (Babesia bovis) en bovinos de la Prepuna de Jujuy, Argentina. Vet. Arg. 34 (351) wwww.veterinariargentina.com

Nava S, Mastropaolo M, Guglielmone AA, Mangold AJ. 2013. Effect of deforestation and introduction of exotic grasses as livestock forage on the population dynamics of the cattle tick Rhipicephalus (Boophilus) microplus (Acari: Ixodidae) in northern Argentina. Res. Vet. Sci. 95: 1046-1054.

Sarmiento N, Zimmer P. 2010. Casuística de babesiosis y anaplasmosis bovina 2009-2010. INTA Mercedes. Ediciones INTA. 456: 4 pp.

Solari MA, Dutra F, Quintana S. 2013. Epidemiología y prevención de los hemoparásitos (Babesia y Anaplasma) en el Uruguay. En: Fiel C, Nari A (Coord.). Enfermedades Parasitarias de Importancia Clínica y Productiva en Rumiantes. Fundamentos epidemiológicos para su diagnóstico y control. Ed. Hemisferio Sur S.R.L, Montevideo. pp. 657-688.

Späth EJA. 1986. Un estudio epidemiológico de babesiosis y anaplasmosis bovina en el Valle de Lerma, provincia de Salta. Rev. Med. Vet. (B. Aires) 67: 274-281. 